\title{
Development of a real-time, semi-capacitive impedance phlebography device
}

\author{
Sören Weyer ${ }^{1,3}$, Hannes Weber ${ }^{1}$, Christian Kleeberg ${ }^{2}$, Steffen Leonhardt ${ }^{1}$, Tobias Wartzek $^{1}$ \\ 1. Chair for Medical Information Technology, Helmholtz Institute, RWTH Aachen University, Pauwelsstr. 20, Aachen, Germany \\ 2. tic Medizintechnik GmbH \& Co. KG, Endelner Feld 9, Dorsten, Germany \\ 3. E-mail any correspondence to: weyer@hia.rwth-aachen.de
}

\begin{abstract}
Chronic venous insufficiency of the lower limbs is a disease which is caused by an increased blood pressure inside the veins of the leg and the resulting increase of the contained blood volume. This work focuses on developing a device which uses impedance plethysmography, also known as impedance phlebography, to obtain information about the blood volume in the lower leg and provides the possibility to measure the impedance semi contact-less, e.g. through compression stockings. Furthermore a real-time beat-to-beat interval detection algorithm was implemented. Finally, the function of the developed impedance measuring system and the whole system was verified by comparing it with a gold standard. In comparison to the conductive coupling, the system performed similarly. The analysis showed that the developed system is suitable for semi-capacitive IPG. The algorithm was implemented conservatively since it provided a good false-positive rate of $0 \%$, but only a moderate sensitivity of about $68 \%$. Reliable and continuous measurement of the pulse signal was only possible in periods of immobility.
\end{abstract}

Keywords: chronic venous insufficiency, impedance plethysmography, capacitive measurement, real-time detection algorithm

\section{Introduction}

One of the most common diseases is the chronic venous insufficiency (CVI) of the lower extremities, which is caused by a permanent pressure overload of the vein. The venous tree is defective, incapable of removing sufficient amounts of blood from the peripheral veins. This causes pooling of blood and intravascular fluid at the lower gravitational parts of the body - the legs.

CVI may affect up to $20 \%$ of adults and as much as $1.5 \%$ to $2 \%$ of the annual healthcare budgets in European countries are spent on the management of CVI [1,2].

Most of the blood in the legs is removed by the contraction of the calf muscle through which pressure is applied to the deep veins, so called muscle pump. If the calf muscles are not used over an extended period of time, the amount of blood in the leg veins increases and a harmful pressure overload occurs. The consequences are, for example, the accumulation of edema or damage of the venous valves, which leads to an increase of the blood pressure, causing additional valves to fail. The most common treatment for CVI is the compression therapy. The compression is usually used by compression stockings where pressure is applied to the deep veins in the legs.
A diagnostic method for detecting CVI is impedance phlebography (IPG), which is a non-invasive medical method that measures small changes of the electrical resistance of the calf. This measurement method was first described by Nyboer [3] and aims at quantitatively determining blood volume changes of a tissue section. Compared to photoplethysmography, this method allows a measurement of the blood volume variation independently of the arterial blood circulation, which can vary due to physiological processes, for example due to ambient temperature [4]. A disadvantage of the IPG is the technical equipment. In IPG, a high-frequent current (typically between $5 \mathrm{kHz}$ and $100 \mathrm{kHz}$ ) is injected into the tissue by two electrodes. Two other electrodes are used to measure the corresponding voltage drop, which depends on the tissue composition. The impedance equals the quotient of the measured voltage and injected current.

The relationship between the measured impedance and changes of blood volume in the measured segment as described by Nyboer [3] is given by:

$$
\frac{\Delta V}{V}=-k \cdot \frac{\Delta Z}{Z}
$$

$V$ is the total volume of the examined tissue, $\Delta V$ is the blood volume change in the segment, $Z$ is the basal impedance, $\Delta Z$ is the blood related impedance change and $k$ is a proportionality constant. This constant $k$ depends on two factors. First, it is determined by the composition of the examined body segment. Second, it depends on the measuring frequency. For example, a proportionality constant of $k \approx 1$ can be assumed for a measurement at the mid-calf and a frequency of $100 \mathrm{kHz}$ [5]. In the lower limbs, the measured change in impedance $\Delta Z$ is mainly a superposition of two hemodynamic processes. One process is the pulsatile flow of the beating heart and the hemodynamic properties of the arteries. The other process is the absolute amount of blood in the veins which causes a further additive component of $\Delta Z$. This addition is mainly visible for changes of the observed body position or during muscle pump tests. Since these processes usually transport a lot of blood, the resulting change of impedance is much higher than the pulsatile component of the heart.

Beside the usual conductive coupling of a wearable bioelectrical impedance measurement, textile integrated impedance cardiography or spectroscopy for personal health care applications is currently under research $[6,7,8]$. An alternative contactless method to measure the impedance of the thoracic region is the use of wearable magnetic induction systems.[9]. 
The aim of this work is to develop a personal healthcare device that can be used while wearing compression stockings and that provides information from impedance plethysmography. The device should be wearable, unobtrusive, should measure the beat-to-beat pulse rate and warn the user when he should use his muscle pump.

\section{Materials and methods}

\section{System concept}

The proposed semi-capacitive impedance phlebography should apply an alternating current by commercial conductive electrodes, but should provide a contact-less measurement through an isolating material of the corresponding voltage drop. In Figure 1 the systematic concept of the semi-capacitive impedance phlebography device is shown.

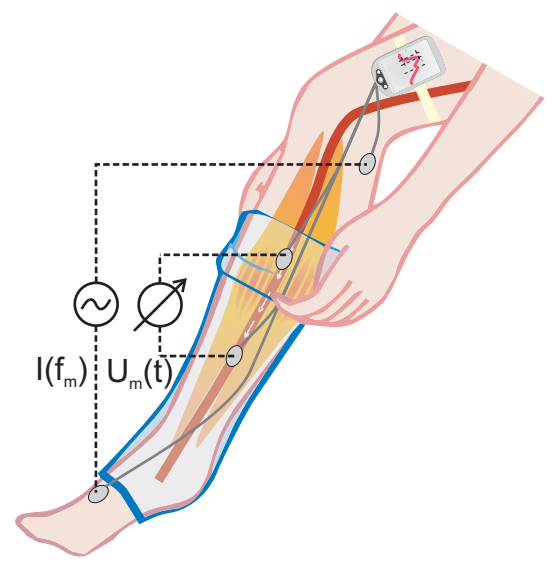

Fig. 1: System concept of the semi-capacitive impedance phlebography device.

It uses a tetra-polar arrangement of electrodes. The fourelectrode technique avoids electrode polarization and minimizes the influence of the skin impedance $[10,11]$. The outer pair of conductive electrodes passes a small alternating current $I_{\mathrm{m}}$ of $0.375 \mu \mathrm{A}_{\mathrm{RMS}}$ at a frequency $f_{\mathrm{m}}$ into the leg and the inner pair of capacitive electrodes measures the resulting time-depending voltage drop $U_{\mathrm{m}}$. The inner electrodes should measure the impedance contact-less trough an isolating material, like pressure stockings.

The system samples the corresponding impedance with the sampling-rate $f_{\mathrm{s}}$ and should provide an integrated, realtime peak-detection of the IPG-signal for further analysis. Furthermore, the high sampling rate should provide the recording of a time-resolved IPG-signal.

Two specifications are essential to build the device: the sample rate and the resolution. The minimal sample rate can be estimated based on the results of Soares Filho et al. [12]. They examined the time derivative of $\Delta Z$ with respect to the spectral frequency composition of the leg impedances of healthy and arteriosclerotic subjects. The study showed that about $99 \%$ of the signal energy in both studied groups was below $32.7 \mathrm{~Hz}$. Since the derivation of spectral components is amplified proportional to their frequency, it can be concluded that the power of $\Delta Z$ is also distributed below this frequency. Therefore, a sample rate of approximately $66 \mathrm{~Hz}$ fulfills the Nyquist-Shannon sampling theorem to reconstruct all relevant information of the IPG-signal. Since the normal heart rate at rest is usually between 60 and 100 beats per minute ( $1 \mathrm{~Hz}$ to $1.67 \mathrm{~Hz}$ ), $66 \mathrm{~Hz}$ provides an oversampling of the heart rate signal [13].

The resolution of the measurement device is an issue since the basal impedance $Z$ is much higher than the amplitude of the pulsatile component $\Delta Z$. For example, a basal impedance of $85 \Omega$ but only a pulsatile component of about $35 \mathrm{~m} \Omega$ can be measured in a segment of $20 \mathrm{~cm}$ of a lower leg [14]. The high basal impedance $Z$ and the small pulsatile component $\Delta Z$ is a challenge for a proper analogue-digital-conversion, unless $Z$ is removed by an analogous filter.

\section{Capacitive electrodes}

Since the device should measure through an isolating material, a capacitive measurement system was developed. The capacitive measuring was done by active electrodes as an impedance converter, which enables a low-impedance processing of the measured potential $\varphi_{\mathrm{G}}$. Figure 2 shows the measurement of a potential $\varphi_{\mathrm{G}}$ with a capacitive electrode. The lower part of the Figure shows the corresponding equivalent circuit.

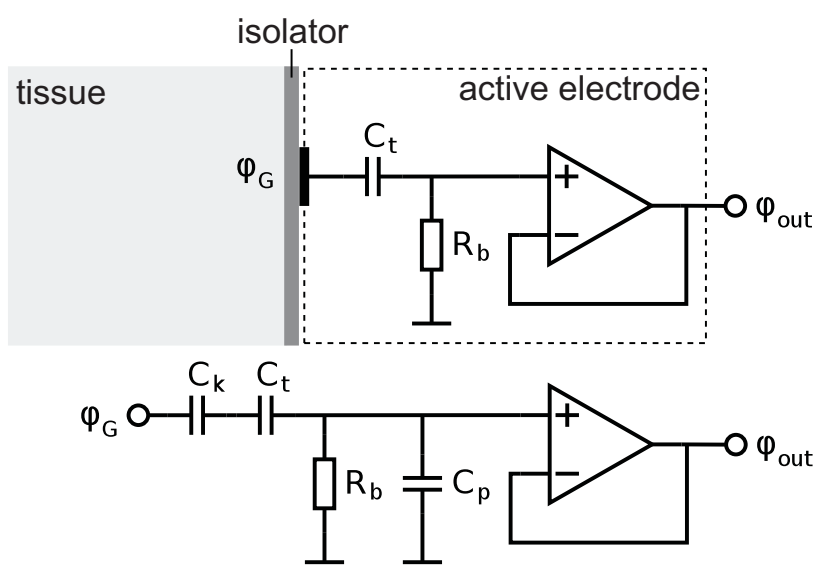

Fig. 2: Measuring arrangement for impedance measurement with two or four electrodes and the corresponding equivalent electric circuit.

The capacitor $C_{\mathrm{k}}$ represents the capacitive coupling between the electrode and the tissue. It is dependent on the surface of the electrode, the distance between the examined tissue, and the dielectric property of the material between the electrode and the measured object. For example, a $0.5 \mathrm{~mm}$ thick cotton layer between electrode and measured object with an electrode area of $16.6 \mathrm{~cm}^{2}$ at a measuring frequency of $200 \mathrm{kHz}$ results in a coupling capacitance of $25 \mathrm{pF}$ to $30 \mathrm{pF}$ [15].

In order to ensure a capacitive coupling in the case of a conductive connection between the electrode surface and the tissue, the capacitor $C_{\mathrm{t}}$ was added. The capacitance $C_{\mathrm{t}}$ should be much larger than $C_{\mathrm{k}}$, so that the latter dominates the resulting capacitance of the series connection of the two 
capacitors. Static charges that may accumulate on the electrode surface, as well as the bias current of the operational amplifier, are discharged by the resistance $R_{\mathrm{b}}$. The capacity $C_{\mathrm{p}}$ summarizes the parasitic capacitances. These may arise through the trace routing of the circuit board. Finally, the operation amplifier provides the low impedance potential $\varphi_{\text {out }}$.

Eq. (2) describes the Laplace transfer function of the active electrode under the assumption of an ideal operational amplifier.

$$
\begin{aligned}
G(s) & =\underbrace{\frac{s R_{\mathrm{b}}\left(C_{\mathrm{k}}^{\prime}+C_{\mathrm{p}}\right)}{s R_{\mathrm{b}}\left(C_{\mathrm{k}}^{\prime}+C_{\mathrm{p}}\right)+1}}_{\begin{array}{c}
\text { capacitive voltage divider } \\
\frac{C_{\mathrm{k}}^{\prime}+C_{\mathrm{p}}}{C_{\mathrm{k}}^{\prime}}
\end{array}}, \\
\text { with } C_{\mathrm{k}}^{\prime} & =\frac{C_{\mathrm{k}} C_{\mathrm{t}}}{C_{\mathrm{k}}+C_{\mathrm{t}}}
\end{aligned}
$$

The active electrodes show high-pass behavior, with a cut-off frequency of

$$
f_{\mathrm{c}, \mathrm{EL}}=\frac{1}{2 \pi R_{\mathrm{b}}\left(C_{\mathrm{k}}^{\prime}+C_{\mathrm{p}}\right)} .
$$

On the one hand, this behavior requires a minimum size of $R_{\mathrm{b}}$, which is dependent on the frequency of the measurement current $I_{\mathrm{m}}$ and the smallest expected value of $C_{\mathrm{k}}$. On the other hand, the high-pass behavior can be used to attenuate the common mode noise caused by the power supply lines through the electrode.

This is particularly useful when the operational amplifier is not only connected as an impedance converter, but also as an amplifier. In this case, the amplified common mode noise can drive the operational amplifier into saturation quickly. Nevertheless, by using the high-pass behavior, the attenuation of the lower frequencies is dependent on the coupling capacitance $C_{\mathrm{k}}$. Eq. (2) also shows that the measured signal is attenuated by the capacitive voltage divider which is formed by $C_{\mathrm{k}}^{\prime}$ and $C_{\mathrm{p}}$. This limits the minimum of the coupling capacitance, in which a measurement is possible.

The schematics are shown in Figure 3.

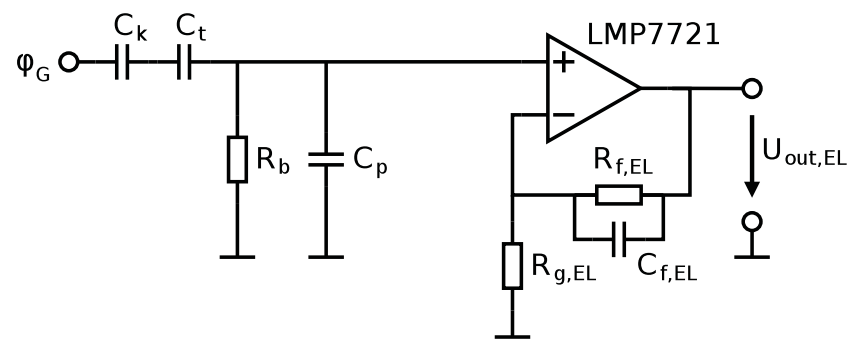

Fig. 3: Circuit diagram of the active electrode.

In this illustration $\varphi_{\mathrm{G}}$ represents the potential at the tissue and the coupling capacitor between tissue and the electrode is denoted by $C_{\mathrm{k}}$. The decoupling capacitor $C_{\mathrm{t}}$, the bias resistor $R_{\mathrm{b}}$ and the parasitic capacitance $C_{p}$ were described above. The measurement electrodes were made from square, double sided, printed circuit boards with a side length of $3 \mathrm{~cm}$, and a sensitive surface on one side. To guarantee a capacitive coupling, the sensitive area was isolated by polypropylene tape. The discrete components of the circuit board were placed on the opposite side of the electrode as shown in Figure 4. The operational amplifier used in the active electrode was the LMP7721 (Texas Instrument, Dallas, USA), which was supplied by a bipolar supply voltage of $\pm 1.8 \mathrm{~V}$. The op-amp was connected in the noninverting amplifier configuration with a bandwidth limitation effectuated by $C_{\mathrm{f}, \mathrm{EL}}$ and $R_{\mathrm{f}, \mathrm{EL}}$. Thus, the transfer function of the active electrode is composed by a constant gain, a highpass and a low-pass, as shown in equation (5).

$$
\begin{aligned}
G_{\mathrm{EL}}(s) & =A_{\mathrm{EL}} G_{\mathrm{HP}, \mathrm{EL}}(s) G_{\mathrm{LP}, \mathrm{EL}}(s) \\
\text { with: } & \\
A_{\mathrm{EL}} & =\left(\frac{C_{\mathrm{k}}^{\prime}}{C_{\mathrm{k}}^{\prime}+C_{\mathrm{p}}}\right)\left(1+\frac{R_{\mathrm{f}, \mathrm{EL}}}{R_{\mathrm{g}, \mathrm{EL}}}\right) \\
& \approx 1+\frac{R_{\mathrm{f}, \mathrm{EL}}}{R_{\mathrm{g}, \mathrm{EL}}}, \text { with } C_{\mathrm{k}}^{\prime} \gg C_{\mathrm{p}} \\
G_{\mathrm{HP}, \mathrm{EL}}(s) & =\frac{s R_{\mathrm{b}}\left(C_{\mathrm{k}}^{\prime}+C_{\mathrm{p}}\right)}{s R_{\mathrm{b}}\left(C_{\mathrm{k}}^{\prime}+C_{\mathrm{p}}\right)+1} \\
& \approx \frac{s R_{\mathrm{b}} C_{\mathrm{k}}^{\prime}}{s R_{\mathrm{b}} C_{\mathrm{k}}^{\prime}+1}, \text { with } C_{\mathrm{k}}^{\prime} \gg C_{\mathrm{p}} \\
G_{\mathrm{LP}, \mathrm{EL}}(s) & =\frac{1}{s R_{\mathrm{f}, \mathrm{EL}} C_{\mathrm{f}, \mathrm{EL}}+1} \\
C_{\mathrm{k}}^{\prime} & =\frac{C_{\mathrm{k}} C_{\mathrm{t}}}{C_{\mathrm{k}}+C_{\mathrm{t}}}
\end{aligned}
$$

To limit the voltage measurement to $f_{\mathrm{m}}$, the following components were chosen: $R_{\mathrm{f}, \mathrm{EL}}=82 \mathrm{k} \Omega, R_{\mathrm{g} . \mathrm{EL}}=39 \mathrm{k} \Omega$, and $C_{\mathrm{f}, \mathrm{EL}}=10 \mathrm{pF}$. Hence, the cutoff frequency of the lowpass filter was $f_{\mathrm{g}, T P, \mathrm{EL}}=194 \mathrm{kHz}$ and, with large coupling capacitances, these values resulted in a gain of $A_{\mathrm{EL}} \approx 3.1$. The remaining components were chosen to $C_{\mathrm{t}}=100 \mathrm{nF}$ and $R_{\mathrm{b}}=10 \mathrm{M} \Omega$. Both active electrodes were connected to the main board by a four wire cable. Two cables were used for the positive and negative operating voltage, one for the ground connection, and one for the output voltage of the active electrode. A photo of the active electrodes and the electrode position is shown in Figure 4.
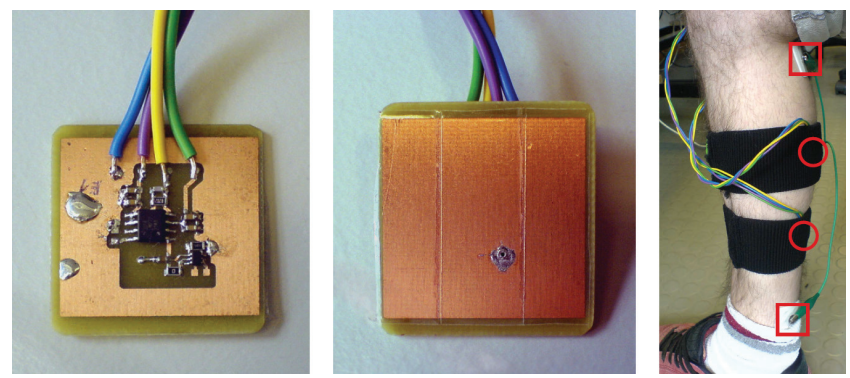

Fig. 4: Upper and lower sides of the active electrode and the measuring arrangement. In the right picture the blue circles mark the positions of the outer current electrodes and the red circles mark the position of the measuring electrodes.

\section{Measurement device}

The impedance measuring system consisted mainly of the AFE4300 chip (Texas Instruments, Dallas, USA) and active electrodes for capacitive voltage measurement, as mentioned 
above. To increase the resolution of the impedance measurement the signal was directly amplified at the active electrodes. An additional amplification was done by a differential amplifier between the electrodes and the AFE4300 chip.

The circuit is shown in Figure 5.

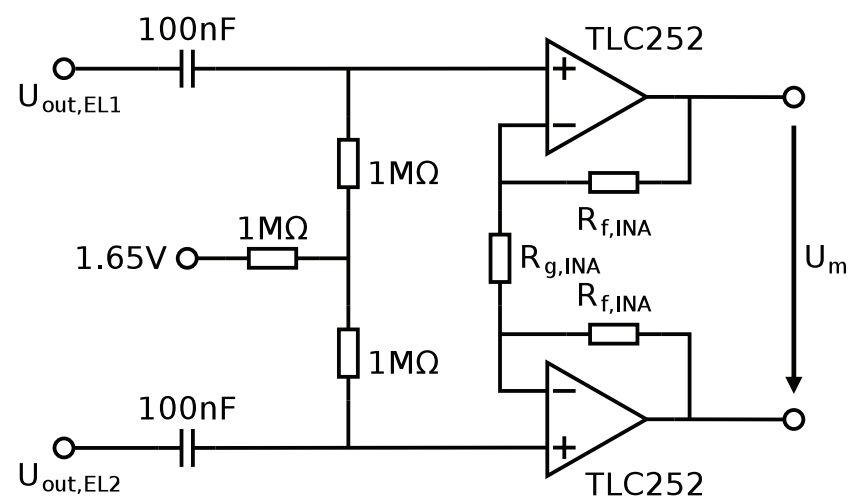

Fig. 5: Circuit diagram of the differential amplifier.

The operational amplifier (TLC252, Texas Instruments, Dallas, USA) and AFE4300 need a supply voltage of $3.3 \mathrm{~V}$. In the given frequency range, the circuit was independent of the frequency of the input signal and the gain can be calculated as

$$
A_{\mathrm{INA}}=\frac{U_{\mathrm{m}}}{U_{\text {out }, \mathrm{EL} 1}-U_{\mathrm{out}, \mathrm{EL} 2}}=1+\frac{2 R_{\mathrm{f}, \mathrm{INA}}}{R_{\mathrm{g}, \mathrm{INA}}} .
$$

By selecting $R_{\mathrm{f}, \mathrm{INA}}=10 \mathrm{k} \Omega$ and $R_{\mathrm{g}, \mathrm{INA}}=892 \Omega$, the gain was $A_{\text {INA }} \approx 23.42$. The voltage $U_{\mathrm{m}}$, which was amplified by the differential amplifier, was used next as measurement voltage of the AFE4300. The full-wave rectification mode of the AFE4300 was used to demodulate the signal. In this paper, a measuring frequency $f_{\mathrm{m}}=24 \mathrm{kHz}$ and a sampling rate of $f_{\mathrm{s}}=64 \mathrm{~Hz}$ was used. The sample rate was slightly below the minimal sampling frequency described above, but in order to achieve a higher oversampling of the Delta-Sigma analog-to-digital converter (ADC) and the reduction of the quantization error, a lower sample rate was selected.

Additionally, the oversampling rate was increased by an increase of the clock frequency $f_{\mathrm{CLK}, \mathrm{AFE}}$ of the AFE4300. Since this oversampling was not intended by the manufacturer, the behavior of the chip was thoroughly tested (the effects of the oversampling on signal quality are given in the appendix) [16]. Due to the change of the clock frequency, the resulting measurement and sampling frequency were changed to

$$
\begin{gathered}
f_{\mathrm{m}}=\frac{k_{\mathrm{div}, \mathrm{AFE}} \cdot f_{\mathrm{CLK}, \mathrm{AFE}}}{1024} \\
f_{\mathrm{s}}=\frac{f_{\mathrm{s}, \mathrm{AFE}} \cdot f_{\mathrm{CLK}, \mathrm{AFE}}}{1 \mathrm{MHz}}
\end{gathered}
$$

Here, $f_{\mathrm{s}, \mathrm{AFE}}$ denotes the configured sampling rate of the AFE4300 and $f_{\mathrm{s}}$ the resulting operating frequency. $k_{\text {div, AFE }}$ denotes an integer which controls a clock divider to generate different measurement frequencies. The normal clock frequency of the AFE4300 is $f_{\mathrm{CLK}, \mathrm{AFE}}=1 \mathrm{MHz}$, but in this paper a cycle of $f_{\mathrm{CLK}, \mathrm{AFE}}=4 \mathrm{MHz}$ was used. In order to smooth the demodulated signal, the datasheet provides the connection of a $10 \mu \mathrm{F}$ capacitor. In this paper, a capacitor of $480 \mathrm{nF}$ was chosen to set the cutoff frequency of the low-pass $f_{\mathrm{g}, \mathrm{demod}}$ to $33 \mathrm{~Hz}$.

\section{Signal processing}

The aim of the signal processing was the extraction and separation of the beat-to-beat heart interval. The signal processing was implemented on a MSP430F2618 microcontroller. First, the inverted measured signal was filtered with a bandpass filter to separate the physiological frequency components. A real-time beat-to-beat interval detection algorithm was implemented to the recorded regularities in the occurrence of the maxima over time in order to distinguish measurement artifacts from heart related pulsations. If there was a high regularity of the detected maxima, the presence of a heart related pulse signal was assumed and the signal processing detected a heartbeat and used the time between the new and last recognized maximum as one heart cycle.

The process of the signal processing is illustrated in Figure 6.

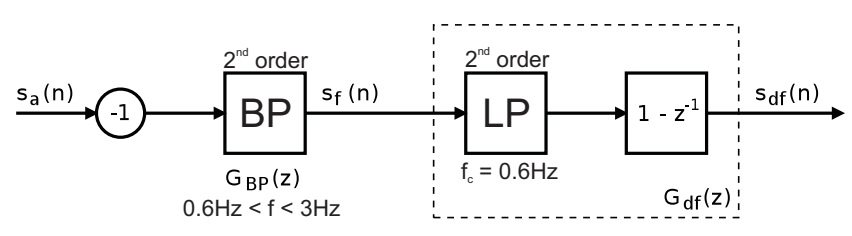

Fig. 6: Filter for detection of maxima of $s_{\mathrm{a}}(n)$.

For the detection of the maxima, two signals were obtained from the measured signal $s_{\mathrm{a}}(n)$. First, the measured impedance values were inverted and the frequency components components below $0.6 \mathrm{~Hz}$ and above $3 \mathrm{~Hz}$ were removed by a second order band-pass. The z-transfer function of the filter and the obtained signal $s_{\mathrm{f}}(n)$ are given by the following equations:

$$
\begin{array}{r}
G_{\mathrm{BP}}(z)=\frac{0.0119-0.0237 z^{-2}+0.0119 z^{-4}}{1-3.64 z^{-1}+5 z^{-2}-3.01 z^{-3}+0.7171 z^{-4}} \\
s_{\mathrm{f}}(n) \propto-\bullet S_{\mathrm{f}}(z)=-S_{\mathrm{a}}(z) G_{\mathrm{BP}}(z)
\end{array}
$$

Further, this signal was smoothed by a second order lowpass with cut-off frequency at $0.6 \mathrm{~Hz}$ and subsequently differentiated. These two operations were done by the filter $G_{\mathrm{df}}(z)$. The z-transfer function of the filter and the obtained signal $s_{\mathrm{df}}(n)$ are given by the following equations:

$$
\begin{array}{r}
G_{\mathrm{df}}(z)=\frac{8.33 \cdot 10^{-4}+8.33 \cdot 10^{-4} z^{-1}}{1-1.92 z^{-1}+0.92 z^{2}}+ \\
\frac{-8.33 \cdot 10^{-4} z^{-2}-8.33 \cdot 10^{-4} z^{-3}}{1-1.92 z^{-1}+0.92 z^{2}} \\
s_{\mathrm{df}}(n) \multimap-\bullet S_{\mathrm{df}}(z)=-S_{\mathrm{f}}(z) G_{\mathrm{df}}(z)
\end{array}
$$

For better illustration, Figure 7 shows an impedance signal measured at the calf as well as the filtered signals $s_{\mathrm{f}}(n)$ and $s_{\mathrm{df}}(n)$. 

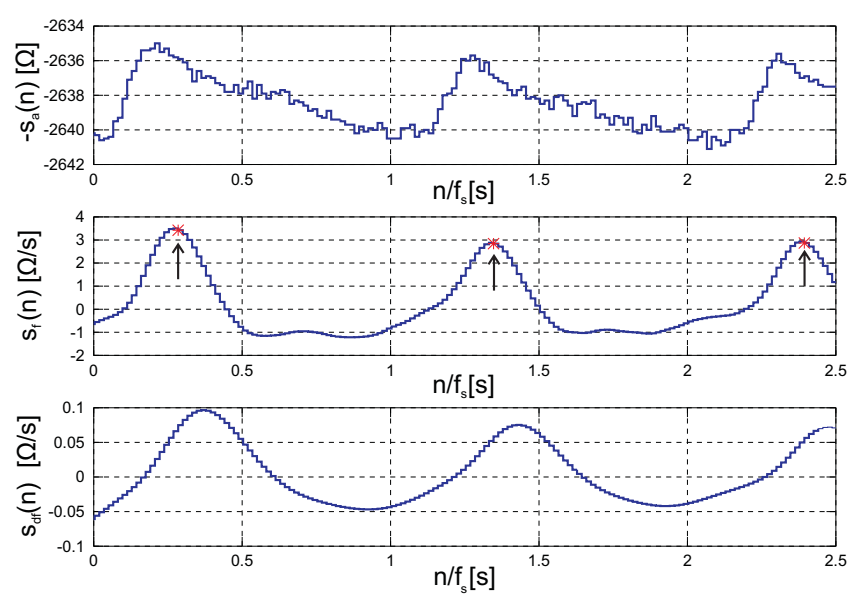

Fig. 7: Measured impedance signal $s_{\mathrm{a}}(n)$ and the filtered signals $s_{\mathrm{f}}(n)$ and $s_{\mathrm{df}}(n)$. The red cross in $s_{\mathrm{f}}(n)$ mark the maxima which are detected by the algorithm.

The pulsatile component in $s_{\mathrm{f}}(n)$ is obvious, but a simple detection of local maxima would also provide points between beats, for example, at the local maximum near $\frac{n}{f_{\mathrm{s}}} \approx 0.7 \mathrm{~s}$. Therefore, $s_{\mathrm{f}}(n)$ was smoothed by a low-pass while the zero crossings of $s_{\mathrm{df}}(n)$ were used to detect the maxima points. Due to the strong smoothing, there was a considerable time delay for the detection of a zero crossing, from positive to negative, in $s_{\mathrm{df}}(s)$. Therefore, the algorithm detected only zero crossings, from negative to positive, in $s_{\mathrm{df}}(n)$. After a zero-crossing was detected, $s_{\mathrm{f}}(n)$ was maintained for the first value, which was smaller than the previous values.

This algorithm showed the best results if flanks rose steadily in $s_{\mathrm{f}}(n)$ and it provided a detection of the maxima with a small and relatively constant delay to $s_{\mathrm{a}}(n)$. The number of samples corresponding to this delay was given by the order of $G_{\mathrm{BP}}(z)$ and one additional sample to detect the decrease in $s_{\mathrm{f}}(n)$. Therefore, the delay time could be calculated to $\frac{5}{f_{\mathrm{s}}} \approx 78 \mathrm{~ms}$, which is small and predictable enough to fulfill the real-time-computing criterion.

\section{Experimental setting for validation test}

For the evaluation, the impedance of the leg was measured and the data were recorded with a PC. In the further analysis, the current $I_{\mathrm{m}}$ was applied to the lower leg by commercial $\mathrm{Ag} / \mathrm{AgCl}$ ECG-electrodes. The electrodes were placed on the rear side of the lower leg, just below the knee and slightly above the ankle. The distance between the measuring electrodes was about $10 \mathrm{~cm}$ and the upper electrode was placed on the calf muscle belly, inclined slightly to the inside of the lower leg. To compare the results with a gold standard, a PPG was measured simultaneously at the second toe of the same leg. For this purpose, a commercially available finger clip device was used (CMS50E, Contec Medical Systems, Qinhuangdao, China). The recordings of the PPG signal were sampled with $60 \mathrm{~Hz}$ and the IPG signal with $64 \mathrm{~Hz}$. For the evaluation two experimental setting as validation test were performed.

The first evaluation step was the analysis of the influence of the capacitive measuring method on the signal quality and the detection of the pulse rate. For this, three different configurations were tested: a conductive coupling, a passive capacitive coupling, and an active capacitive coupling. In order to acquire a conductive coupling to the tissue, conventional ECG-adhesive electrodes were used. For passive capacitive coupling, the same electrodes as for the active coupling (described above) were used, but in this case the circuit-boards were unequipped. To provide three comparable conditions, the gain of the active electrode was set to $A_{\mathrm{EL}}=1$ by removing the resistor $R_{\mathrm{g}, \mathrm{EL}}$. Furthermore, the gain resistor of the differential amplifier was raised to $R_{\mathrm{g}, \mathrm{INA}}=470 \Omega$, resulting in a gain of $A_{\mathrm{INA}} \approx 43.6$. Hence, the resulting gain of the active electrode and differential amplifier was reduced to $63 \%$ of the original value, which should be considered for the comparison to the next results.

In the three configurations, the lower leg was stretched on a chair and the signal was recorded for $95 \mathrm{~s}$. For comparison with the gold standard, the PPG-signal was recorded simultaneously on the second toe. Each peak time $t_{\mathrm{PPG}}(i)$ of the PPG signal was allocated to a peak time $t_{\mathrm{IPG}}(j)$ in the IPG signal using the rule

$$
\left|t_{\mathrm{PPG}}(i)-t_{\mathrm{IPG}}(j)\right|<\lambda=\lambda_{\text {rel }} \frac{60}{H F_{\mathrm{PPG}}(i)} .
$$

The parameter $\lambda_{\text {rel }}$ was used to adapt the factor to the corresponding heart rate and was chosen as $\lambda_{\text {rel }}=0.3$.

During the next test, the subject sat on a chair while the examined lower leg was bent at an angle of about $90^{\circ}$ to the thigh and placed perpendicular to the ground to the ground. Additionally to the second test, the leg was stretched and laid on the seat surface of a second chair. To prevent the occurrence of major motion artifacts during the measurements, the subject tried to move as little as possible and was breathing normally. From these measurements the pulsations were separated in correct and wrong detected beats compared to the control results of the gold standard. The accuracy of the detection algorithm, with respect to the peak time and the heart rate, was analyzed in more detail.

Since the measured PPG signal was artifact free, the beats were identified by the local maxima. From the PPG, the peak time $t_{\mathrm{PPG}}(i)$ and the corresponding heart rate $H R_{\mathrm{PPG}}(i)$ $\left[\mathrm{min}^{-1}\right]$ were extracted. The number of detected heartbeats, precisely peak times, were denoted as $N_{\mathrm{PPG}}$. The peak time $t_{\mathrm{IPG}}(j)$ and heart rate $H R_{\mathrm{IPG}}$, which were extracted by the algorithm, were denoted as $N_{\mathrm{IPG}}$.

\section{Results}

In the first evaluation step no false-positive pulse beats were found by the detection algorithm. Thus, the false-positive rate was $0 \%$ for all three experiments. The sensitivity of the conductivity measurement method was approximately $65 \%$. In this experiment, the capacitive active electrodes could achieve a sensitivity of about $34 \%$. The lowest sensitivity was found for the capacitive passive measurement, which was only $10 \%$. By analysis of the raw data pulse curve, it 
was apparent that the conductive measurement was not so prone to movement artifacts, like breathing, as the capacitive measurements has been. The impedance signal of the capacitive passive measurement was additionally superimposed of strong common-mode interference.

The second evaluation step showed that the waveform of the IPG signal strongly varied over the time. This behavior possibly correlated with the breathing of the subject, which is reflected in form of a low-frequency, additive component in the PPG signal. It also showed that the pulsations of the IPG signal occurred simultaneously with those of the PPG signal and the expected time delay of $78 \mathrm{~ms}$ was not recognizable. This was explainable by the time delay of the signal processing of the PPG-meter or it might be a physiologically time delay of the pulse wave transit time between calf and toe.

Every beat of the PPG was assigned to the IPG by the wellknown criterion of eq. (17). By this method, each element of $t_{\mathrm{IPG}}(j)$ which was detected by the algorithm could be assigned to the according $t_{\mathrm{PPG}}(i)$. On the downside, not each peak time of $t_{\mathrm{PPG}}(i)$ could be found in $t_{\mathrm{IPG}}(j)$. Therefore, the elements of $t_{\mathrm{PPG}}(i)$ were classified to true-positive $T P(k)$, and false-negative $F N(l)$. The number of elements $N_{T P}, N_{F P}$ and $N_{F N}$ describe the number of true-positive, false-positive and false-negative findings.

Tab. 1: Measurement period and number of detected pulse waves for the bent and stretched leg.

\begin{tabular}{rrrrrrr}
\hline & period & $N_{\text {PPG }}$ & $N_{\text {IPG }}$ & $N_{\text {TP }}$ & $N_{F N}$ & $N_{F P}$ \\
\hline bent leg & $128 \mathrm{~s}$ & 150 & 97 & 97 & 53 & 0 \\
stretched leg & $100 \mathrm{~s}$ & 116 & 81 & 81 & 35 & 0 \\
\hline
\end{tabular}

Table 1 shows the measurement period, the amount of heart beats which are detected by the gold standard, and true-positive false-positive and false-negative findings of the algorithm in the IPG signal. The sensitivity of the new system $T P R=\frac{N_{\mathrm{TP}}}{N_{\mathrm{PPG}}}$ was $65 \%$ for the bent leg and $70 \%$ for the stretched leg. Since the algorithm did not detect false heart beats, there were no false-positive findings. Therefore, the false-positive rate $F P R=\frac{N_{\mathrm{FP}}}{N_{\mathrm{PPG}}}$ was $0 \%$ in both cases.

These results are comparable to the results of the previous section for the conductive measurement. For the evaluation of the detected heart rate a Bland-Altman plot was used to illustrate the performance, as shown in Figure 8 for the stretched leg and Figure 9 for the bent leg. The horizontal lines are drawn at the mean difference and at the limits of agreement. These are defined as the mean difference plus and minus 2 times the standard deviation $\sigma$ of the differences.

In the case of the bent leg, most differences of the heart rates $\Delta H R_{T P}(k)=H R_{T P, \mathrm{PPG}}(k)-H R_{T P, \mathrm{IPG}}(k)$ were between $-2 \min ^{-1}$ and $2 \mathrm{~min}^{-1}$, with an average difference slightly below $-0.2 \mathrm{~min}^{-1}$. The evaluation showed for the stretched leg that $90 \%$ of $H R_{T P}$ were between $\pm 1 \mathrm{~min}^{-1}$ with an average difference of $0.2 \mathrm{~min}^{-1}$.

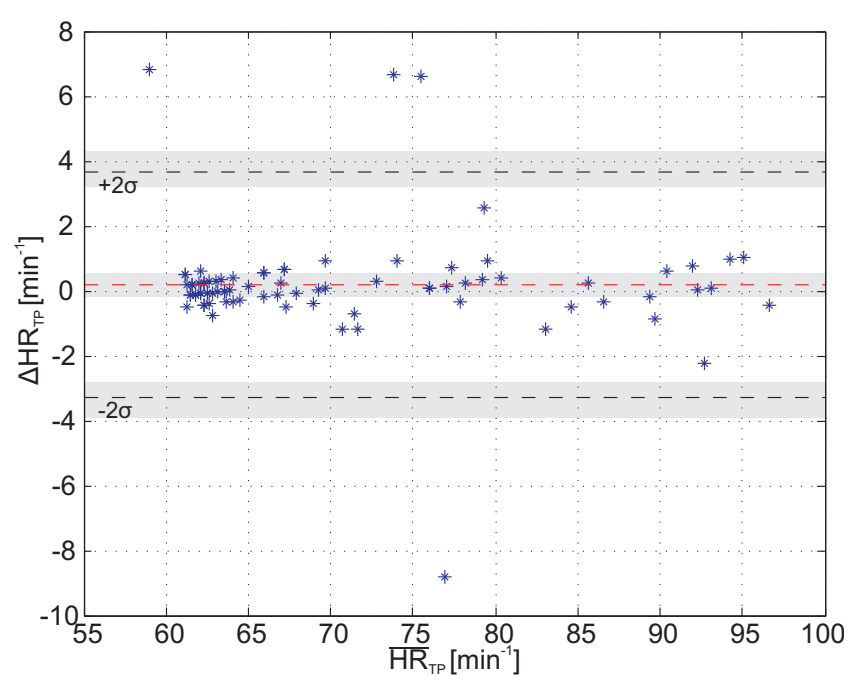

Fig. 8: Bland-Altman plot of the new measurement device and the PPG as gold standard of the stretched leg.

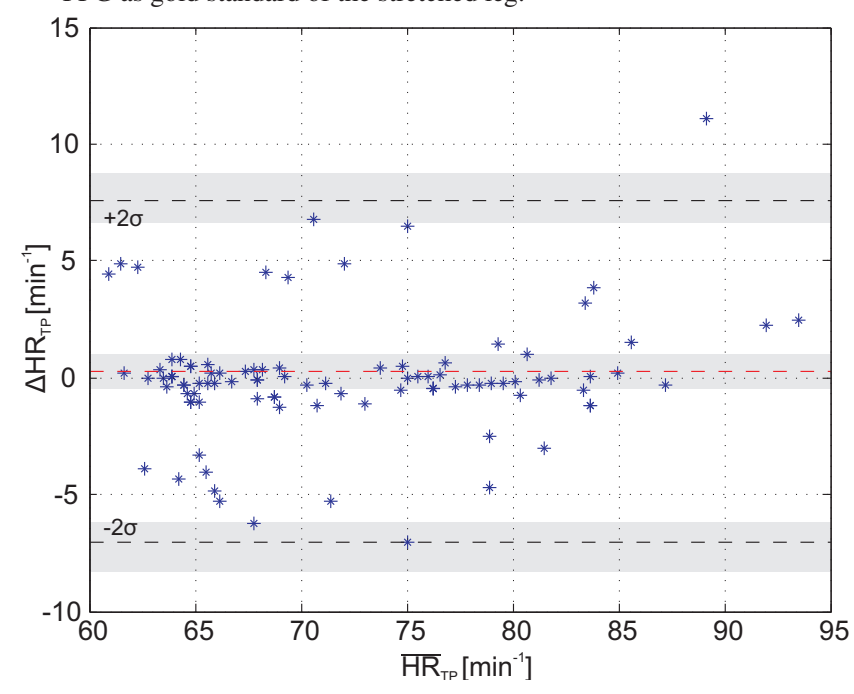

Fig. 9: Bland-Altman plot of the new measurement device and the PPG as gold standard of the bent leg.

\section{Discussion and Conclusion}

This work proposes an integrated solution for monitoring the health status of risk patients as well as the according procedure to design and construct a wearable, semi-capacitive system.

Firstly, the analog front end and the digital back end of the measurement system were described. For this purpose, the frequency response was mathematically derived. Based on this derivation, the analog design of the capacitive electrodes and the amplifier circuit were shown. The system also provides the possibility to replace the capacitive active electrodes with conventional ECG electrode pads in order to increase the signal quality, resulting in a more reliable detection of pulsations. The digital back end is based on the microcontrollers AFE4300 and MSP430. The standard wiring, which was given by the manufacturer, was modified to fulfill the requirements concerning the measurement accuracy. In this work, the measurement system was used to record the time-varying impedance of a leg, which is related to blood.

Secondly, a real-time algorithm for the detection of beatto-beat pulse waves was presented. The change of the fre- 
quencies of the heartbeat from conventional clinical measurement modalities (such as ECG) are a well-studied problem, e.g. for heart rate variability analysis [17]. Furthermore, the real-time analysis of a beat to beat interval by means of IPG gives the advantage that another physiological rhythm can be evaluated contactless, even while wearing compression stockings. Hence, many useful parameters can be extracted in combination with a wearable ECG system, as for example the rise time of the IPG signal or the real-time pulsewave velocity.

Thirdly, a proof-of-concept trial was conducted to evaluate the whole system in vivo. Two validation tests were performed. The first evaluation step was the analysis of the influence of conductive as well as passive, and active capacitive coupling regarding signal quality and the detection of the pulse rate. The false-positive rate was $0 \%$ for all three experiments. The sensitivity was the highest during the conductive measurement and about twice as large as during the active capacitive measurement. The passive capacitive electrodes, compared to the other two, were of insufficient quality, since the measurements were superimposed by many artifacts and had a high common mode noise.

The second evaluation step was done with the highest amplification of the active capacitive electrodes. The analysis showed that the algorithm was implemented conservatively; it provided a low false-positive rate of $0 \%$, but only a moderate sensitivity of about $68 \%$. In any case, a reliable and continuous measurement of the pulse signal was only possible in periods of immobility. Furthermore, strong breathing deteriorated the detection rate of the algorithm. The analysis also showed that the results were different for both body postures while the measurment with stretched leg showed slightly better results.

The evaluation suggests that a semi-capacitive impedance phlebography is a promising method to measure blooddependent time-varying changes of the impedance. Therefore, such a system could be used for personal-healthcare or telemonitoring applications.

The evaluation showed that a semi-capacitive impedance phlebography is a promising method to measure blooddependent time-varying changes of the impedance. However, different measurement positions on the leg yield differences in the signal quality, which requires further investigation. Even in commercial IPG devices, the electrode positioning is not yet standardized, as it is for impedance cardiography. Nevertheless, the system could assist the physician in continuously diagnosing and monitoring the peripheral vascular status of the patient, who is wearing compression stockings. Furthermore, valuable information about the reaction of the vascular system following its manipulation with, e.g., tilt table tests, exercise, or vasoactive drugs, could be gained. Not only clinical, but also home applications are conceivable. Therefore, such a system could be used for personal healthcare or telemonitoring applications. The system could provide information about the fitting of compression stockings or the current blood flow in the legs. Further investigations in these directions seem appropriate and necessary.

\section{Acknowledgment}

This work has been supported by the German Federal Ministry of Economics and Technology (BMWi) under the grant ZIM (AIF FK KF2561908AK3, 'RheoStim').

\section{Appendix}

To investigate the effects of the oversampling on signal quality, the differential amplifier was bridged and the AFE4300 was connected directly and without calibration to a $1.8 \mathrm{k} \Omega$ resistor. Between the measurements the clock frequency of the AFE4300 with $f_{\mathrm{CLK}, \mathrm{AFE}}=1 \mathrm{MHz}$ was increased up to $4 \mathrm{MHz}$ and the configuration was changed in order to receive the resulting sampling rate as $f_{\mathrm{s}}=64 \mathrm{~Hz}$, independent of the clock frequency. For each configuration 150 samples were taken as shown in the comparison in Figure 10.

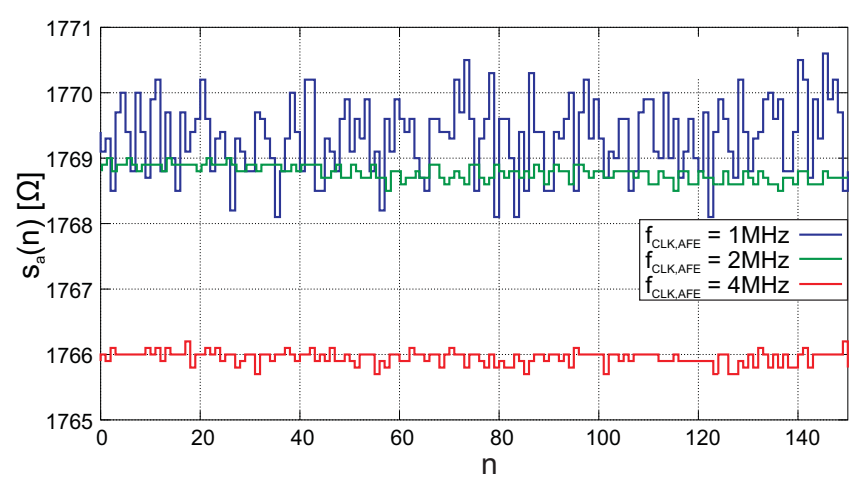

Fig. 10: Measured signal $s_{\mathrm{a}}(n)$ of a $1.8 \mathrm{k} \Omega$ resistance at $f_{\mathrm{s}}=64 \mathrm{~Hz}$, $f_{\mathrm{s}}=24 \mathrm{kHz}$ and varying clock frequencies $f_{\mathrm{CLK}, \mathrm{AFE}}$.

Since the system was not calibrated, the absolute value of the measurement was irrelevant, but the noise was an interesting quality index for the analysis of different clock frequencies. It is apparent that the measurements with a clock frequency of $2 \mathrm{MHz}$ and $4 \mathrm{MHz}$ were more precise than $1 \mathrm{MHz}$. The standard deviations for $f_{\mathrm{CLK}, \mathrm{AFE}}=1 \mathrm{MHz}$ was about $530 \mathrm{~m} \Omega$ and for the other two frequencies $100 \mathrm{~m} \Omega$. It is also apparent that the mean value of the measured resistance decreases with increasing operating frequency.

\section{References}

1. Ruckley CV. Socioeconomic impact of chronic venous insufficiency and leg ulcers. Angiology. 1997;48(1):67-69. http://dx.doi.org/10.1177/000331979704800111.

2. Simka M, Majewski E. The social and economic burden of venous leg ulcers: focus on the role of micronized purified flavonoid fraction adjuvant therapy. American Journal of Clinical Dermatology. 2003;4(8):573-581. http://dx.doi.org/10.2165/00128071-200304080-00007.

3. Nyboer J, Kreider MM, Hannapel L. Electrical Impedance Plethysmography: A Physical and Physiologic Approach to Peripheral Vascular Study. Circulation. 1950;2(6):811-821. http://dx.doi.org/10.1161/01.CIR.2.6.811. 
4. Schüler R. Neue methodische Ansätze zur Objektivierung von Diagnostik und Therapiekontrolle bei venösen Insuffizienzen der unteren Extremitäten. Ilmenau: ISLE; 2001.

5. Schüler R. Apparative Gefäßdiagnostik: Untersuchungsmethoden und deren Anwendung. Ilmenau: ISLE; 1998.

6. Ulbrich M, Mühlsteff J, Sipilä A, Kamppi M, Koskela A, Myry M, et al. The IMPACT shirt: textile integrated and portable impedance cardiography. Physiological Measurement. 2014;35(6):1181-1196. http://dx.doi.org/10.1088/0967-3334/35/6/1181.

7. Schlebusch T, Röthlingshöfer L, Saim K, Köny M, Leonhardt S. On the Road to a Textile Integrated Bioimpedance Early Warning System for Lung Edema. In: Body Sensor Networks (BSN), 2010 International Conference on; 2010. p. 302-307. http://dx.doi.org/10.1109/BSN.2010.21.

8. Márquez JC, Seoane F, Lindecrantz K. Textrode functional straps for bioimpedance measurements-experimental results for body composition analysis. European journal of clinical nutrition. 2013;67 Suppl 1:S22-7.

http://dx.doi.org/10.1038/ejcn.2012.161.

9. Teichmann D, Kuhn A, Leonhardt S, Walter M. The MAIN Shirt: a textile-integrated magnetic induction sensor array. Sensors (Basel, Switzerland). 2014;14(1):1039-1056. http://dx.doi.org/10.3390/s140101039.

10. Foster KR, Lukaski HC. Whole-body impedance-what does it measure? The American Journal of Clinical Nutrition. 1996;64(3 Suppl):388S-396S.
11. Rosell J, Colominas J, Riu P, Pallas-Areny R, Webster JG. Skin impedance from $1 \mathrm{~Hz}$ to $1 \mathrm{MHz}$. IEEE Transactions on Biomedical Engineering. 1988;35(8):649-651. http://dx.doi.org/10.1109/10.4599.

12. Soares Filho W, Brum OF, Panerai RB. Spectral analysis of electrical impedance measurements on the lower limbs. IEEE Transactions on Biomedical Engineering. 1983;30(7):387391. http://dx.doi.org/10.1109/TBME.1983.325038.

13. Berkow R. The Merck manual of medical information. New York: Pocket; 2000.

14. Nyboer J. Electrical Impedance Plethysmography - The Electrical Resistive Measure of the Blood Pulse Volume, Peripheral and Central Blood Flow. Ch. C. Thomas; 1970.

15. Eilebrecht B, Willkomm J, Pohl A, Wartzek T, Leonhardt S. Impedance measurement system for determination of capacitive electrode coupling. IEEE Transactions on Biomedical Circuits and Systems. 2013;7(5):682-689. http://dx.doi.org/10.1109/TBCAS.2013.2237905.

16. Texas Instruments. Datasheet - AFE4300 - Low-Cost, Integrated Analog Front-End for Weight-Scale and Body Composition Measurement. Dallas, Texas, USA;

17. Köhler BU, Hennig C, Orglmeister R. The principles of software QRS detection. IEEE engineering in medicine and biology magazine : the quarterly magazine of the Engineering in Medicine \& Biology Society. 2002;21(1):42-57. http://dx.doi.org/10.1109/51.993193. 